\title{
A Comparative Study of Dynamic Hip Screw Versus Proximal Femoral Nailing in Surgical Management of Trochanteric Fractures in a Tertiary Care Centre, Thrissur District, Kerala
}

\author{
Abino D.J. ${ }^{1}$, Krishnakumar C.V. ${ }^{2}$ \\ 1, 2 Department of Orthopaedics, Government Medical College, Thrissur, Kerala, India.
}

\section{ABSTRACT}

\section{BACKGROUND}

Intertrochanteric fractures account for approximately half of the hip fractures in the elderly. Safe and efficient restoration of mobility is the goal of the treatment of intertrochanteric fractures. At the same time, we need to reduce the medical complications, and finally restore the patient to the pre-operative status. Dynamic hip screw gives good results but is sometimes not suitable particularly in unstable intertrochanteric fractures. The objective of this study was to compare dynamic hip screw and proximal femoral nail with respect to their functional and radiological outcomes during surgical management of the trochanteric fracture.

\section{METHODS}

A prospective study was conducted among 100 patients admitted with trochanteric fracture, in Government medical college, Thrissur, out of which 50 patients underwent fixation with proximal femoral nail and 50 with dynamic hip screw. Using proforma, socio-demographic details, details of admission, mode of trauma, details of fracture and surgery, complications and Harris hip score were assessed, minimum follow up was 6 months following surgery and longest follow up was 12 months.

\section{RESULTS}

Functional outcome was assessed with Harris hip score, and of the patients treated with dynamic hip screw, $28 \%$ had a good score, whereas in patients treated with proximal femoral nail $42 \%$ had good score (P-value 0.019), a significant difference was found between duration of non-weight bearing and type of fixation, also there was a significant difference in Harris Hip Score (P-value of 0.0001)

\section{CONCLUSIONS}

The proximal femoral nail (PFN) group had better functional outcomes both radiologically and clinically.

\section{KEY WORDS}

Intertrochanteric Fractures, Proximal Femoral Nail, Dynamic Hip Screw.
Corresponding Author: Dr. Krishnakumar C.V., Associate Professor, Department of Orthopaedics, Government Medical College, Thrissur, Kerala, India.

E-mail: drkrishnakumarcv@gmail.com

DOI: $10.14260 / \mathrm{jemds} / 2021 / 807$

How to Cite This Article:

Abino DJ, Krishnakumar CV. A comparative study of dynamic hip screw versus proximal femoral nailing in surgical management of trochanteric fractures in a tertiary care centre, Thrissur district, Kerala. J Evolution Med Dent Sci 2021;10(45):3998-4002, DOI: 10.14260/jemds/2021/807

Submission 02-09-2021,

Peer Review 10-09-2021,

Acceptance 21-12-2021,

Published 31-12-2021.

Copyright (C) 2021 Abino D.J. et al. This is an open access article distributed under Creative Commons Attribution License [Attribution 4.0 International (CC BY 4.0)] 


\section{BACKGROUND}

The incidences of trochanteric fractures are also found to be increasing due to higher longevity and rising incidence of road traffic accidents.

In older adults, hip fractures can lead to severe morbidity and mortality. Thrombosis, muscle atrophy, pulmonary infection, and bedsores can be prevented by allowing the patients to get out of the bed earlier and also by promoting early mobilization.

The various treatment options for trochanteric fractures are operative and non-operative. In the early 19th century when the operative technique was not evolved enough to do a stable fixation, the non-operative method used to be the treatment of choice. Non-operative treatment can only be considered in chronic dementia or non-ambulatory patients who are experiencing pain which is controllable with analgesics and rest, or in patients with terminal diseases with a life expectancy of fewer than 6 weeks, unresolved medical comorbidities that prevent surgical treatment, also in active infectious diseases which are a contraindication for insertion of a surgical implant. Non-operative treatment may be indicated in incomplete pertrochanteric fractures diagnosed by MRI. Non-operative approaches include reduction via traction and early mobilization within the limits of pain tolerance. ${ }^{1}$

There is a high complication rate in the conservative approach. The increased incidence of varus deformity and shortening results in poor function.

Various operative treatment modalities have been introduced to date for the reduction of trochanteric fractures, including dynamic hip screw, bipolar hemiarthroplasty, proximal femoral nail, trochanteric fixation nail (TNF) and external fixation, all of which have their specific advantages and disadvantages. ${ }^{2}$

DHS has been considered as the standard fixation for extracapsular femoral fracture and yielded good results in patients with stable intertrochanteric fractures. ${ }^{3}$

Dynamic hip screw requires a larger exposure, greater tissue trauma and anatomical reduction. All these increase morbidities. It has a greater probability of infection and also involves significant blood loss. It also causes varus collapse leading to shortening and inability of the implant to survive until the fracture union. 4

A plate and screw device may weaken the bone mechanically. The common causes of fixation failure are instability of the fractures, osteoporosis, lack of anatomical reduction, failure of fixation device and incorrect placement of the screw. ${ }^{5}$

Intramedullary nailing has become a popular method of stabilization of intertrochanteric fractures in adults. ${ }^{6}$ A nail itself gives support to the posteromedial wall and resists excessive collapse. For good outcomes and reducing the risk of complications, near-anatomical reduction and optimal positioning of implants are of paramount importance. Some pitfalls like implant failure do occur in the proximal femoral nail (PFN). This may be due to specific unbalanced biomechanical forces acting on an implant at and around the hip joint. Implant failure is a common complication of PFN surgery. This may be due to the back out of screws, cut through of implant through bone, "Z" effect, and "reverse Z" effect, or even breakage of the PFN.
Currently, patients with trochanteric fractures attending our hospital are treated either with DHS or PFN based on the choice of surgeon.

\section{Objectives}

- To compare the functional and radiological outcome of dynamic hip screw versus proximal femoral nailing in the surgical management of trochanteric fracture in patients treated at Government Medical College, Thrissur.

- To determine which implant would provide the best results with the least complications.

\section{METHODS}

A prospective study was conducted among 100 patients admitted with trochanteric fracture, in the Department of Orthopaedics, Government medical college Thrissur, from $16 / 4 / 2018$ to $30 / 4 / 2019$. Out of them, 50 patients who underwent fixation with PFN and 50 with DHS were selected. Using proforma, socio-demographic details, details of admission, mode of trauma, details of fracture and surgery, complications, and Harris Hip Score at the end of 6 months up to 1 year following surgery were assessed. Finally, data were coded and entered in Microsoft Excel and also analysed using IBM SPSS software version 16.

\section{Study Tools}

Proforma containing questions on socio-demographic details (age, gender, economic status), mode of trauma, type of trochanteric fracture, associated with any other injury, type of fixation, radiological findings, Harris Hip Score, complications, and comorbidities.

Anteroposterior and mediolateral radiographs of hip at immediate postoperative and at follow up visit.

Harris hip score with maximum 100 Points (Score interpretation 90 -100 - Excellent, 80-89 - Good, 70-79 - Fair, $<70$ - Poor)

\section{Sample Size}

- According to a study by Kumar et al. on functional outcome of intertrochanteric fracture after fixation with PFN or DHS a comparative study, excellent functional outcome with Harris Hip Score was found in $56.5 \%$ in case of those who had surgery with PFN and $36.67 \%$ in those who had surgery with DHS.7

- The minimum sample size required in each group was 49.62, so a sample of 50 was taken in each group.

- $\quad$ The total sample size was 100 .

\section{Inclusion Criteria}

- Age $>18$ years.

- All types of trochanteric fractures treated with either dynamic hip screw or proximal femoral nails who had given consent. 


\section{Exclusion Criteria}

- Previous surgery of proximal femur.

- Pathological fractures other than osteoporosis.

- Ongoing chemotherapy or irradiation treatment due to malignancy.

- Other associated fractures of the lower limb.

- Pre-existing femoral deformity.

- Screw cut off phenomenon during surgery.

\section{Data Management and Statistical Analysis}

Data was appropriately coded and entered in Microsoft Excel after the completion of data collection. Analysis of the data was done using the software statistical package for social sciences (SPSS) version 16.0.

Mean and standard deviation were calculated for all quantitative variables. Normally distributed data means of two groups were compared using Student's t test. The Mann Whitney test was used for skewed data or scores. Proportions were compared using chi-square test or Fisher's exact test whichever was applicable. Qualitative or categorical variables were described as frequencies and proportions. P-values less than 0.05 were considered statistically significant.

\section{RESULTS}

The mean age of subjects treated by PFN was 62.76. The mean age of patients treated by DHS was 68.18. Most of the patients were between 61 and 80 years in both groups. The youngest patient was 21-year-old and the oldest was 89 years.

PFN group included $58 \%$ males and $42 \%$ females while DHS group included $48 \%$ males and $52 \%$ females.

In this study, the majority of study subjects $76 \%$ belonged to the below poverty line (BPL) in the DHS group, whereas in the PFN group $82 \%$ belonged to BPL.

In patients treated with DHS, 30 (60\%) were right-sided fractures and $20(40 \%)$ were left-sided, whereas in the PFN group, 34 (68\%) were right-sided fractures and $16(32 \%)$ left-sided.

In patients treated with DHS, $56 \%$ injuries were due to domestic falls, $44 \%$ were due to road traffic accidents whereas in the PFN group, $74 \%$ were due to domestic falls and $26 \%$ due to road traffic accidents.

In patients who were operated by DHS, 38 (76 \%) were stable intertrochanteric fractures, $12(24 \%)$ were unstable intertrochanteric fractures whereas in PFN group, 24 (48 \%) were stable intertrochanteric fractures, 17 (34\%) were unstable intertrochanteric and $9(18 \%)$ were subtrochanteric.

Harris hip score was calculated 6 months after surgery.

Harris hip score grading: less than 70 points-poor; 70-79 points -fair; 80-89 points- good; $90-100$ points-excellent.

In this study, in patients treated with DHS, $28 \%$ had good scores, $60 \%$ had fair scores and $12 \%$ had poor scores, whereas in patients treated with PFN, $42 \%$ had good scores, $56 \%$ had fair scores and $2 \%$ had a poor score.

It was found that there was a significant difference in Harris hip score between DHS and PFN fixation with MannWhitney Test, at $Z$ value -2.339 , at a $P$ value of 0.019
In patients treated with DHS, $12 \%$ had shortening, whereas in those treated with PFN, $4 \%$ had shortening, this was not found to have a significant association with a chisquare value of 2.174 at P-value- 0.269

In patients treated with DHS, majority (62\%) needed 2 months of non-weight bearing, whereas in those treated with PFN, majority (64\%) needed 1 month of non-weight-bearing, which was found to be statistically significant by independent sample T-test, with a T value of 4.184 , at a P value of 0.0001

In patients treated with DHS, majority (50\%) needed 1 month, $48 \%$ needed 2 months, and $2 \%$ needed 3 months of weight-bearing with the walker, whereas in patients treated with PFN, $56 \%$ needed 1 month, $42 \%$ needed 2 months, and $2 \%$ needed 3-month weight-bearing with a walker which was not found to be statistically significant by independent sample T-test, at a P value of 0.582

In patients treated with DHS, majority (72\%) needed 1 month, $28 \%$ needed 2 months weight bearing with support, whereas in patients treated with PFN, $64 \%$ needed 1 month, $36 \%$ needed 2 months weight-bearing with support which was not found to be statistically significant by independent sample T-test, at P-value of 0.396

In both groups majority (96\%) of patients didn't have any kind of infection after surgery

In patients treated with DHS, $12 \%$ had varus malunion, whereas in those treated with PFN, $4 \%$ had varus malunion. But this difference was not found to be significant with a chisquare value of 2.174 at a P-value of 0.140 .

In patients treated with DHS, $16 \%$ had rotational deformity whereas in those treated with PFN, $6 \%$ had a rotational deformity. But this difference was not found to be significant with a chi-square value of 2.554 at a P-value of 0.110 .

In patients treated with DHS, none of the patients had non-union whereas in case of PFN, $2 \%$ had non-union, but this difference was not found to be significant with a chisquare value of 1.010 at a P-value of 0.315 .

None of the study subjects had screw cut out and Z phenomenon during surgery and follow up.

It was found that there was no significant difference between neck-shaft angle between DHS and PFN fixation by Independent Sample T-test, at a P-value of 0.541.

\section{DISCUSSION}

In the 1950s, the management of unstable intertrochanteric fractures was revolutionized by the development of DHS. Dynamic hip screw (DHS) soon became the implant of choice. This was due to the favourable results and low rate of complications of DHS. DHS provides controlled compression at the fracture site. The use of DHS has been supported by its biomechanical properties which have been assumed to improve the healing of the fracture.

\section{Problems with DHS}

Larger exposure increased operative time, increased blood loss, excessive collapse with shortening and mechanical failures made it unsuitable for unstable fractures. The common causes of fixation failure are instability, 
osteoporosis, improper anatomical reduction, failure of the fixation device and improper placement of the head screw. DHS also needs an intact lateral cortex.

In recent years, PFN introduced by the AO/ASIF group in 1998, has gained much popularity for the treatment of trochanteric fractures. The advantage of PFN fixation is that it is a load-bearing implant, provides a more biomechanically stable construct by reducing the distance between hip joint and implant.?

Reduced lever arm, less telescoping and prevention of medialisation of the shaft are the advantages of PFN.

In a study by Mahesh Kumar et al. the most common age group was 61 to 80 years, with a mean age of 72.23 years. ${ }^{8}$ Gallagher et al. 1980 reported an eightfold increase in trochanteric fractures in men over the age of 80 and women over 50 years of age. ${ }^{9}$ In the study by Gourav single et al. the average age was 67 years. ${ }^{10}$

All trochanteric fractures are not alike. Irrespective of the fixation device, stable trochanteric fractures usually heal well. Unstable trochanteric fractures are associated with complications. Approximately half of the hip fractures in the elderly are intertrochanteric fractures; out of this, more than $50 \%$ of fractures are unstable.

The goal of treatment of any intertrochanteric fracture is to restore early mobility to minimize the risk of medical complications and restore the patient to preoperative status. ${ }^{6}$ Allowing the patients to get out of the bed earlier and promoting early mobilization is necessary to prevent complications such as deep vein thrombosis, bedsores, pulmonary infection, and muscle atrophy. ${ }^{11}$

Cleveland et al.12 pointed out that there are higher incidences of multiple fractures, as of the same or opposite side, which may occur on different occasions. This is due to the underlying instability and inherent weakness of bone structure of the elderly which predisposes them to injuries. After early fixation of such fractures and subsequent early mobilization, these patients could gain a full range of movement at an early date with minimal loss of productivity.

In this study, the PFN group included $58 \%$ males and 42 $\%$ females while the DHS group included $48 \%$ males and 52 $\%$ females. Cleveland et al. in their study had $87.7 \%$ of female patients. They had given the following explanations for their observations:

a. Females have a slightly wider pelvis with a tendency to have coxa vara.

b. They are usually less active and are more prone to senile osteoporosis.

H. B. Boyd and L. L. Griffin ${ }^{13}$ in their study of 300 cases found a marked sex difference. $226(75.8 \%)$ of the patients were females and 74 (24.2\%) were males and the study by St. Urnier K. M., Dresing K. (1995) ${ }^{14}$ suggested that pertrochanteric fractures ordinarily appear to women 10-15 years later than in men.

Most of our patients were above 50 years. Most of the causes were domestic falls (fall at home). Trivial trauma was the usual reason behind the fracture. Most of them were due to osteoporotic fractures. Whereas in young patients they were mainly due to road traffic accidents. In patients treated with DHS, $56 \%$ injuries were due to domestic falls, whereas in the PFN group, $74 \%$ were due to domestic falls.
In a study by Cumming and Nevitt in $1994,{ }^{6}$ the following factors were enumerated for the increased incidence of trochanteric fractures in the elderly.

1. Inadequate protective reflexes, to reduce the energy of fall below a certain critical threshold.

2. Inadequate local shock absorbers like muscle and fat around the hip.

3. Inadequate bone strength at the hip on account of osteoporosis or osteomalacia.

Keneth J. Koval and Joseph D. Zuckerman (1996) ${ }^{15}$ observed that $90 \%$ of hip fractures in the elderly resulted from a simple fall. Hip fractures in young adults were observed usually due to high energy trauma such as road traffic accidents or a fall from height.

In a study by Hassan et al. in the DHS group, $80 \%$ were intertrochanteric fractures, $20 \%$ were subtrochanteric whereas in PFN group, $55 \%$ were intertrochanteric fractures and the remaining $45 \%$ were subtrochanteric. ${ }^{16}$

According to Mervyn Evans, intertrochanteric fractures can be divided into stable or unstable depending upon the integrity of the posteromedial cortex. Fractures with intact posteromedial cortex are considered stable fractures while fractures with loss of posteromedial cortex are considered unstable fractures. Posteromedial cortex constitutes mainly the lesser trochanter. ${ }^{17}$

In study by Anmol Sharma et al. ${ }^{18}$ the mean Harris hip score at 60 months in the DHS group was 88.7 and in the PFN group was 82.2 , with no significant difference in both groups. In a study by Dr Ajithswamy et al. Harris Hip score was significantly more among the PFN group when compared to the DHS group.

\section{CONCLUSIONS}

In our study, we concluded that there is a significant difference in functional outcome of intertrochanteric fractures treated by DHS and PFN from 6 months to 1 year following surgery. Early mobilization is better by PFN.

Data sharing statement provided by the authors is available with the full text of this article at jemds.com.

Financial or other competing interests: None.

Disclosure forms provided by the authors are available with the full text of this article at jemds.com.

\section{REFERENCES}

[1] Kumar R, Singh RN, Singh BN. Comparative prospective study of proximal femoral nail and dynamic hip screw in treatment of intertrochanteric fracture femur. J Clin Orthop Trauma 2012;3(1):28-36.

[2] Kyle RF, Wright TM, Burstein AH. Biomechanical analysis of the sliding characteristics of compression hip screws. J Bone Joint Surg Am 1980;62(8):1308-14.

[3] Zarattini G, Breda L, Zacharia M, et al. Intra-pelvic migration of sliding hip screw during osteosynthesis of hip fracture: a rare avoidable intraoperative complication. J Orthop Case Rep 2015;5(3):25-8. 
[4] Evans EM. The treatment of trochanteric fractures of the femur. J Bone Joint Surg Br 1949;31B(2):190-203.

[5] Gadegone WM, Salphale YS. Proximal femoral nail - an analysis of 100 cases of proximal femoral fractures with an average follow up of 1 year. Int Orthop 2007;31(3):403-8.

[6] Megas P, Kaisidis A, Zouboulis P, et al. Comparative study of the treatment of pertrochanteric fractures-trochanteric gamma nail vs. proximal femoral nail. $\mathrm{Z}$ Orthop Ihre Grenzgeb 2005;143(2):252-7.

[7] Jensen JS, Tøndevold E, Sonne-Holm S. Stable trochanteric fractures. A comparative analysis of four methods of internal fixation. Acta Orthop Scand 1980;51(1-6):811-6.

[8] Kumar NBM, Mahesh U, Kumar GS. A comparative study of proximal femoral fracture fixation with proximal femoral nail and Dynamic hip screw \& plating. Int J Orthop Sci 2017;3(1):499-505.

[9] Gallagher JC, Melton LJ, Riggs BL, et al. Epidemiology of fractures of the proximal femur in Rochester, Minnesota. Clin Orthop Relat Res 1980;(150):163-71.

[10] Singla G. A comparative study of 70 cases of intertrochanteric fracture femur treated with dynamic hip screw and proximal femoral nailing. Int J Res Orthop 2017;3(2):293-7.

[11] Ozkan K, Türkmen I, Sahin A, et al. A biomechanical comparison of proximal femoral nails and locking proximal anatomic femoral plates in femoral fracture fixation: a study on synthetic bones. Indian J Orthop 2015;49(3):347-51.

[12] Menezes DFA, Gamulin A, Noesberger B. Is the proximal femoral nail a suitable implant for treatment of all trochanteric fractures? Clin Orthop Relat Res 2005;439:221-7.

[13] Griffin JB. The calcar femorale redefined. Clin Orthop Relat Res 1982;(164):211-4.

[14] Stürmer KM, Dresing K. Pertrochanteric fractures. Zentralbl Chir 1995;120(11):862-72.

[15] Yadav S, Srivastava DC, Shukla M. Comparative evaluation of dynamic hip screw and proximal femoral nail for fracture of intertrochanteric femur. Int J Res Orthop 2016;2(4):286-90.

[16] Ahmed HH, Bassiooni HAM, Mohamady EM, et al. A comparison study of proximal femoral nail and dynamic hip screw devices in unstable trochanteric fractures. Benha Medical Journal 35(3):413-8.

[17] Koval KJ, Aharonoff GB, Rokito AS, et al. Patients with femoral neck and intertrochanteric fractures. Are they the same? Clin Orthop Relat Res 1996;(330):166-72.

[18] Sharma A, Sethi A, Sharma S. Treatment of stable intertrochanteric fractures of the femur with proximal femoral nail versus dynamic hip screw: a comparative study. Rev Bras Ortop 2017;53(4):477-81. 